Cinémas

Revue d'études cinématographiques

Journal of Film Studies

\title{
Fragments orphiques dans Hiroshima mon amour de Marguerite Duras et d'Alain Resnais
}

\section{Joëlle Cauville et Josette Déléas}

Volume 9, numéro 2-3, printemps 1999

Les Scénarios fictifs

URI : https://id.erudit.org/iderudit/024792ar

DOI : https://doi.org/10.7202/024792ar

Aller au sommaire du numéro

Éditeur(s)

Cinémas

ISSN

1181-6945 (imprimé)

1705-6500 (numérique)

Découvrir la revue

Citer cet article

Cauville, J. \& Déléas, J. (1999). Fragments orphiques dans Hiroshima mon amour de Marguerite Duras et d'Alain Resnais. Cinémas, 9(2-3), 159-173.

https://doi.org/10.7202/024792ar
Résumé de l'article

Comme Orphée, la Française d'Hiroshima mon amour a droit à une seconde chance, à vivre un second amour. Comme Orphée, elle devra renoncer à cet amour, en prenant conscience de l'oubli universel dans lequel elle engloutira Nevers et Hiroshima. Toutefois, cette prise de conscience du personnage incarné par Emmanuelle Riva, loin de se solder par un échec, débouche sur une liberté nouvelle : elle retrouve le goût de l'amour interdit, car elle est redevenue disponible. Cette évolution constitue un exemple de réécriture du mythe au féminin, réécriture qu'Alain Resnais ne trahit pas dans son interprétation cinématographique du scénario de Marguerite Duras. 


\title{
Fragments orphiques dans Hiroshima mon amour de Marguerite Duras et d'Alain Resnais
}

\author{
Joëlle Cauville et Josette Déléas
}

\section{RESUMÉ}

Comme Orphée, la Française d'Hiroshima mon amour a droit à une seconde chance, à vivre un second amour. Comme Orphée, elle devra renoncer à cet amour, en prenant conscience de l'oubli universel dans lequel elle engloutira Nevers et Hiroshima. Toutefois, cette prise de conscience du personnage incarné par Emmanuelle Riva, loin de se solder par un échec, débouche sur une liberté nouvelle: elle retrouve le goût de l'amour interdit, car elle est redevenue disponible. Cette évolution constitue un exemple de réécriture du mythe au féminin, réécriture qu'Alain Resnais ne trahit pas dans son interprétation cinématographique du scénario de Marguerite Duras.

\section{ABSTRACT}

Like Orphée, the French woman of Hiroshima mon amour deserves a second chance, the experience of a second love. Like Orphée, she will have to renounce this love while becoming conscious of the universal oblivion to which she dooms Nevers and Hiroshima. Yet this realization on the part of Emmanuelle Riva's character, far from ending in failure, opens on to a new freedom as she recovers an aptitude for forbidden love thanks to her newfound openness. This evolution is an example of a rewriting of the myth in the feminine, a rewriting; which Alain Resnais does justice to in his filmic interpretation of Marguerite Duras's script. 
Analyser, d'un point de vue mythocritique, Hiroshima mon amour (1959), fruit de la collaboration entre le cinéaste Alain Resnais et l'écrivaine Marguerite Duras, permet de rendre compte de la qualité particulièrement poétique de l'œuvre abordée. La polyvalence du concept de mythe, son caractère ondoyant et plurivoque s'adaptent parfaitement à sa complexité. Lévi-Strauss définissait le mythe comme «une recherche du temps perdu» (1962, p. 225). Or la présence insistante du thème du passé et de la mémoire, l'aspect fondamental, universel des liens d'amour et de mort qui unissent les deux protagonistes de l'histoire constituent à eux seuls une perspective mythique.

Avant de justifier la présence de fragments orphiques dans Hiroshima mon amour, il semble utile de préciser que notre analyse prendra en considération à la fois le film et le texte du scénario tel que nous l'a livré l'édition de 1960, texte second, dépendant, "texte-film».

Ce scénario est précédé d'un synopsis et suivi d'un commentaire intitulé "Les évidences nocturnes (Notes sur Nevers)». Madeleine Borgomano définit ainsi ce texte inclassable: "Cet objet composite inaugure une écriture nouvelle, écriture qui, d'une certaine façon, se refuse à être une écriture, mais ne parvient pas tout à fait à s'abolir elle-même, écriture qui voudrait s'ouvrir aux images et aux sons, devenir elle-même filmique " (p. 36).

Notre seconde précaution est de rappeler que le symbole et le mythe sont le lieu de l'ouverture, du foisonnement des sens, de la complémentarité. L'auteur du Dictionnaire des mythes littéraires, Pierre Brunel, après avoir résumé les trois grandes étapes du mythe orphique - "Orphée et les Argonautes", "Orphée et Eurydice» et enfin "Orphée et les bacchantes '» - , souligne combien le deuxième épisode du récit mythique, celui qui nous intéressera au premier chef, a été privilégié par la littérature, comment, en fait, le mythe littéraire redouble le mythe initial proprement dit, c'est-à-dire l'étape de la quête de la Toison d'or. Il en vient à généraliser son propos par cette mise en garde concernant toute entreprise mythocritique: 


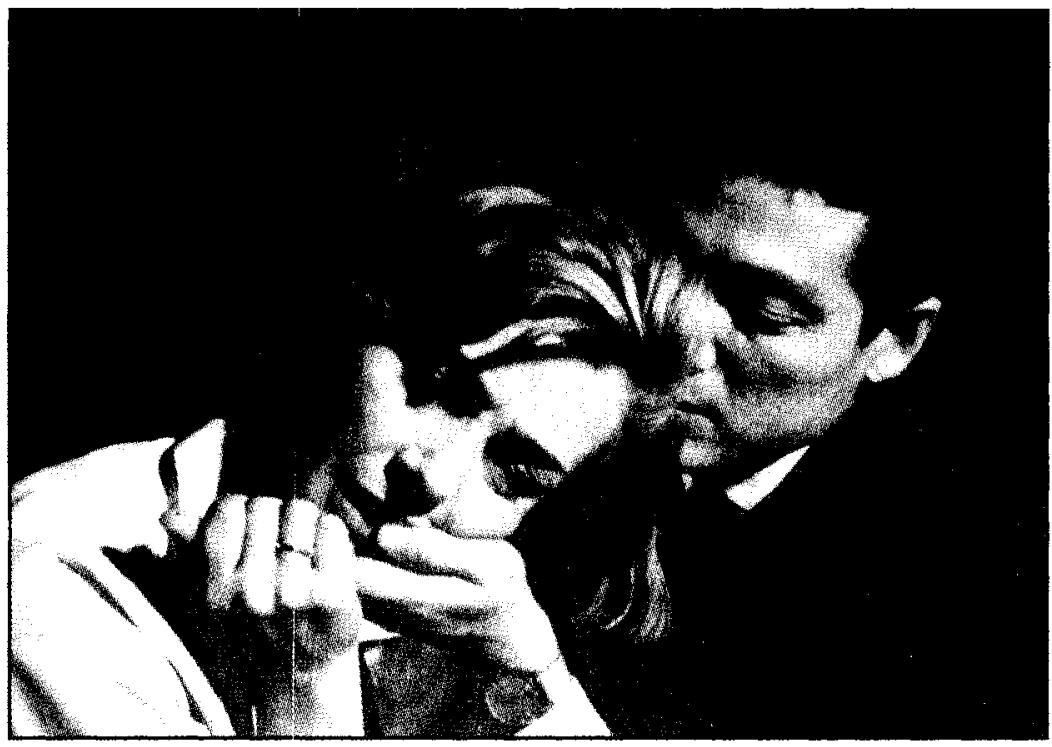

\section{Hiroshima mon amour d'Alain Resnais (1959)}

Mais le mythe, à lui seul, est suffisamment obscur, car il est un lieu de contradictions. L'aventure même dont il propose le récit ne se retrouve pas dessinée dès l'origine, elle s'organise tardivement à partir d'un conglomérat de traditions diverses où il est difficile de faire part des éléments authentiques et de ceux qui se sont introduits par contamination (p. 1095).

De plus, bien qu'Hiroshima mon amour soit indéniablement l'œuvre d'Alain Resnais, il n'en subsiste pas moins que son fondement, son ancrage sont l'accomplissement d'une femme, Marguerite Duras, l'auteure du scénario. Nous avons donc affaire ici à une réécriture du mythe au féminin qui pose inévitablement la question du sexe de la mythologie, et il semble important de rappeler que beaucoup d'écrivaines, surtout contemporaines, dénoncent l'interprétation patriarcale des grands mythes de l'humanité. Toutefois, loin de rejeter le mythe comme moyen d'expression au féminin, elles en forgent des interprétations nouvelles.

Le scénario d'Hiroshima mon amour peut se résumer comme suit: "Quelques heures de la vie d'un couple adultère à Hiroshima

Fragments orphiques dans Hiroshima mon amour de Marguerite Duras et d'Alain Resnais 
en août 1957." Elle, une actrice française d'une trentaine d'années, est venue jouer dans un film international sur la paix. Lui est un architecte japonais. Hiroshima, qui fut le théâtre de l'extrême horreur, est faite à présent à la taille de l'amour. Où était la Française juste avant que la bombe explose sur Hiroshima le 4 août 1945 ? À Nevers, dans la Nièvre, où elle vivait un amour de jeunesse impossible et bouleversant: maîtresse d'un soldat allemand tué à la Libération, elle fut tondue par la foule, enfermée dans une cave par ses parents morts de honte et abandonnée, en proie à la folie. Le souvenir de ce drame reflue à sa mémoire tandis que le Japonais l'écoute et suscite le souvenir. Le temps d'une confidence, le temps d'en vivre, le temps d'en mourir, et l'oubli font son ouvre. Ne subsistent que les noms emblématiques des deux villes qui deviennent les noms des personnages:

ELLE

Hi-ro-shi-ma. C'est ton nom.

Ils se regardent sans se voir. Pour toujours.

LUI

[...] ton nom à toi est Nevers. Ne-vers-en-France (p. 124).

Dans ce néoromantisme que propose Hiroshima mon amour, où s'enchevêtrent les thèmes de l'amour et de la mort, de la mémoire et de l'oubli, comment déceler des traces d'orphisme? Peut-on distinguer certains éléments des trois phases du mythe classique, à savoir la katàbasis, la descente aux enfers; l'anàbasis, le retour, le retournement et la perte; et enfin le sparàgmos, le démembrement? Les deux premières phases appartiennent au second récit mythique, "Orphée et Eurydice" tandis que le troisième a trait à l'épisode d' "Orphée et les bacchantes", le troisième mythème.

Riva, la Française, partage avec Orphée un pouvoir de séduction indéniable. Dans «Les évidences nocturnes", Duras la décrit comme étant "plus séduisante que belle» (p. 154). De plus, elle attire le soldat allemand par la musique: «[...] je sus qu'un homme m'avait écoutée jouer du piano pour la première fois de ma vie» (p. 145). Quant au Dictionnaire des mythes littéraires, il souligne qu' " [...] Orphée est le musicien par excel- 
lence et qu'il se révèle en chacun des traits de sa légende comme le séducteur, à tous les niveaux du cosmos et du psychisme" (p. 711-712). Comme dans le cas d'Orphée, l'être aimé est ravi à la Française au moment où leur amour était le plus fort : "Il me parla de la Bavière où je devais le retrouver. Où nous devions nous marier" (Duras, p. 147). La mythologie classique insiste également sur le bref bonheur d'Orphée avec Eurydice: " La noce à peine achevée, comme la jeune épousée marchait avec ses demoiselles d'honneur dans une prairie, une vipère la mordit au pied et elle mourut" (Hamilton, p. 121). Ainsi, comme pour l'Orphée de la légende, "le veuf, l'inconsolé", aimer pour la Française devienclra très vite "aimer un mort" et sa première katàbasis interviendra lorsque, malade de douleur devant cet absolu d'horreur et de bêtise que représentent l'assassinat de son premier amour en toute légalité (il est ennemi de la France) et le déshonneur public, elle descend au cour de la folie, dans la cave de Nevers.

Dans cette cave, lieu privilégié de la déraison, l'héroïne réapparaît à intervalles réguliers, en proie à son désir forcené de ranimer le souvenir de l'amant mort. Il est une scène, particulièrement saisissante, où, pour se faire du bien, la Française s'écorche les mains qu'elle lèche ensuite. Elle espère ainsi retrouver la saveur du sang de l'Allemand mort, ce sang auquel elle avait goûté, allongée sur son cadavre durant un jour et une nuit: "[...] je peux dire que je n'arrivais pas à trouver la moindre différence entre ce corps mort et le mien... Je ne pouvais trouver entre ce corps et le mien que des ressemblances... hurlantes» (p. 100). Julia Kristeva s'interroge sur la nature nécrophile de la passion de Riva, hypothèse corroborée par Duras elle-même qui dans "Les évidences nocturnes" écrit: "Elle n'en peut plus d'avoir envie de lui, mort» (p. 136). Selon l'auteure de Soleil noir, dépression et mélancolie, cet univers infernal de la cave de Nevers où la famille de Riva la fait passer pour morte, entraîne " [...] une folie, meurtrie et meurtrière (elle est folle de méchanceté, elle crache au visage de sa mère) [qui] ne serait rien d'autre que l'absorption par Elle de sa mort à Lui " (p. 241). Elle s'appuie en cela sur le commentaire de Duras qui écrit: "On pourrait la croire morte tellement elle se meurt de sa mort à lui » (p. 125). 
La folie, les désirs suicidaires de Riva sont ses seuls moyens de garder un lien avec le mort, de ne pas l'oublier, de le récupérer.

Des années plus tard, au cours de sa seconde descente aux enfers, la Française superposera l'histoire d'amour japonaise à l'histoire de Nevers pour revivre cette dernière qu'elle avait refoulée dans l'abîme de l'inconscient. Pour en figurer les profondeurs mal éclairées, et évoquer du même coup la cave obscure, Resnais noie l'espace filmique dans des éclairages sombres où se distinguent à peine le Japonais et la Française. Or, il faut rappeler que dans une perspective psychanalytique, "[...] l'enfer est l'état de la psyché qui a succombé aux monstres dans sa lutte, soit qu'elle ait essayé de les refouler dans l'inconscient, soit qu'elle ait accepté de s'identifier à eux dans une perversion consciente» (Chevalier et Gheerbrant, p. 405).

Dans cette seconde descente chez Hadès, la katàbasis n'a plus uniquement pour objet les retrouvailles avec l'amant mort, mais plutôt le sauvetage de la petite Française morte d'amour à Nevers, que le passé douloureux émergeant jusqu'à la conscience libère et à laquelle il redonne le goût de l'amour impossible. La quête de cette deuxième descente est donc la connaissance de soi.

La seconde phase du mythe: l'anàbasis paraît être l'élément orphique le plus pertinent, le plus développé dans Hiroshima mon amour.

Dans une entrevue de Marguerite Duras accordée à la série télévisée Biographies et diffusée en 1994, l'écrivaine associait étroitement la venue à l'écriture à la notion de tabou, d'interdit. Elle prenait l'exemple de L'Amant, roman autobiographique et prix Goncourt 1984, et celui d'Hiroshima mon amour où, dans les deux cas, un interdit était enfreint: dans l'un, le tabou d'une relation amoureuse entre une mineure blanche et un Chinois est exposé sur toile de fond colonialiste; dans l'autre, l'enjeu est, selon elle, beaucoup plus grave, car la liaison entre le soldat allemand ennemi de la France et la jeune Française entraîne la mort du premier et le déshonneur public de la seconde. Or, le mythe d'Orphée est souvent présenté comme le mythe de l'interdit: celui du regard porté sur le monde de l'au-delà : "Orphée est l'homme qui a violé l'interdit et osé regarder l'invisible» (Chevalier et Gheerbrant, p. 713). 
Dès le début du scénario d'Hiroshima mon amour, le regard est central dans la relation qui s'établit entre les deux amants. Au-delà du choc érotique que suscite l'échange de leurs regards viennent se greffer deux théories différentes du regard, énoncées dans les répliques liminaires du film:

\author{
LUI \\ Tu n'as rien $v u$ à Hiroshima. Rien \\ [...]. \\ ELLE \\ J'ai tout vu. Tout (p. 22).
}

Aux yeux du Japonais, l'affirmation de la Française, qui repose sur une connaissance rationnelle, intellectuelle du drame d'Hiroshima, est révoltante. Le regard qu'elle porte sur le peuple japonais sacrifié est un regard qui chosifie les malades qu'il happe au passage tandis que la caméra agressive fonce sur eux à l'hôpital. Ce regard glacial, dont la froideur est soulignée par des éclairages crus, est mis en accusation par les victimes qui l'arrêtent dans son élan, en fixant à leur tour l'objectif de la caméra ou en s'en détournant. Il est sacrilège comme l'est toute évocation d'Hiroshima parce que Hiroshima même est sacrilège. Dans le texte-film, Marguerite Duras souligne qu'il est impossible de parler d'Hiroshima. Tout ce qu'on peut faire, c'est de parler de l'impossibilité d'en parler. Mais ce que le Japonais ignore encore, c'est que l'expérience de son amante à Nevers, à la fin de la Seconde Guerre mondiale, lui permet d'appréhender l'horreur et l'absurdité de la guerre et de communier avec la souffrance du peuple japonais ${ }^{2}$.

Les didascalies de Marguerite Duras et l'œil de la caméra de Resnais insistent sur le fait que le spectateur / la spectatrice ne voit jamais la Française regarder Hiroshima: "On ne la voit jamais en train de voir" (p. 23).

Ce regard intellectuel que le Japonais interprète comme la négation du regard a été très bien explicité par un critique, W. R. Robinson dans son article intitulé "If You Don't See, You Are Dead ». Il écrit à propos de la Française :

Despite her having come to Hiroshima to look closely, the French woman had no disposition to do so, never 
learned to see while she was there, and did, indeed, as her lover claimed, see nothing. She violates the criterion of seeing that she herself admits to be the key of truth and value by never looking into what is going on about her (p. 27).

"Never looking into what is going on about her." Ceci nous amène à la deuxième conception du regard, que nous qualifierions d' "affectif». C'est ce regard-là qui déclenchera le processus du souvenir et de l'oubli. Or, si le regard que la Française affirme porter sur Hiroshima est une violation d'interdit pour le Japonais, Hiroshima pouvant être comparé à l'enfer orphique, le regard qu'il la forcera à porter sur son passé et à verbaliser sera une violation pour elle qui s'était promis de ne jamais raconter, même pas à son mari, l'amour de Nevers.

Pourtant, alors que le mythe d'Orphée rend le héros coupable de porter un regard sur l'être aimé avant sa sortie des enfers, la trame narrative d'Hiroshima mon amour rend la protagoniste coupable de ne pas regarder son amour passé, elle met en scène une femme en divorce total avec elle-même, faute de ne pas se regarder, si ce n'est dans ses rêves. Ainsi avoue-t-elle: "Nevers, tu vois, c'est la ville du monde, et même c'est la chose du monde à laquelle, la nuit, je rêve le plus. En même temps que c'est la chose du monde à laquelle je pense le moins" (p. 58). Contrairement au mythe traditionnel, regarder en arrière, se regarder, descendre dans les profondeurs de l'inconscient, vont devenir un processus libérateur.

Ainsi le regard affectif se manifeste-t-il à plusieurs reprises au cours du scénario et au lieu d'entraîner la perte de l'être aimé, il est synonyme de renaissance de l'amour ou de son souvenir conscient.

Dans la seconde partie, la Française pose son regard sur la main de son amant japonais endormi et elle occasionne ainsi le premier retour en arrière dans le passé (p. 43). Resnais et Duras font ici un usage cinématographique du mécanisme proustien de la mémoire involontaire qui se déclenche accidentellement grâce à la présence d'un objet, d'une odeur, d'une attitude qui dévoilent soudainement, dans le présent, tout un pan du passé avec les incidents, les émotions qui lui sont rattachés. 


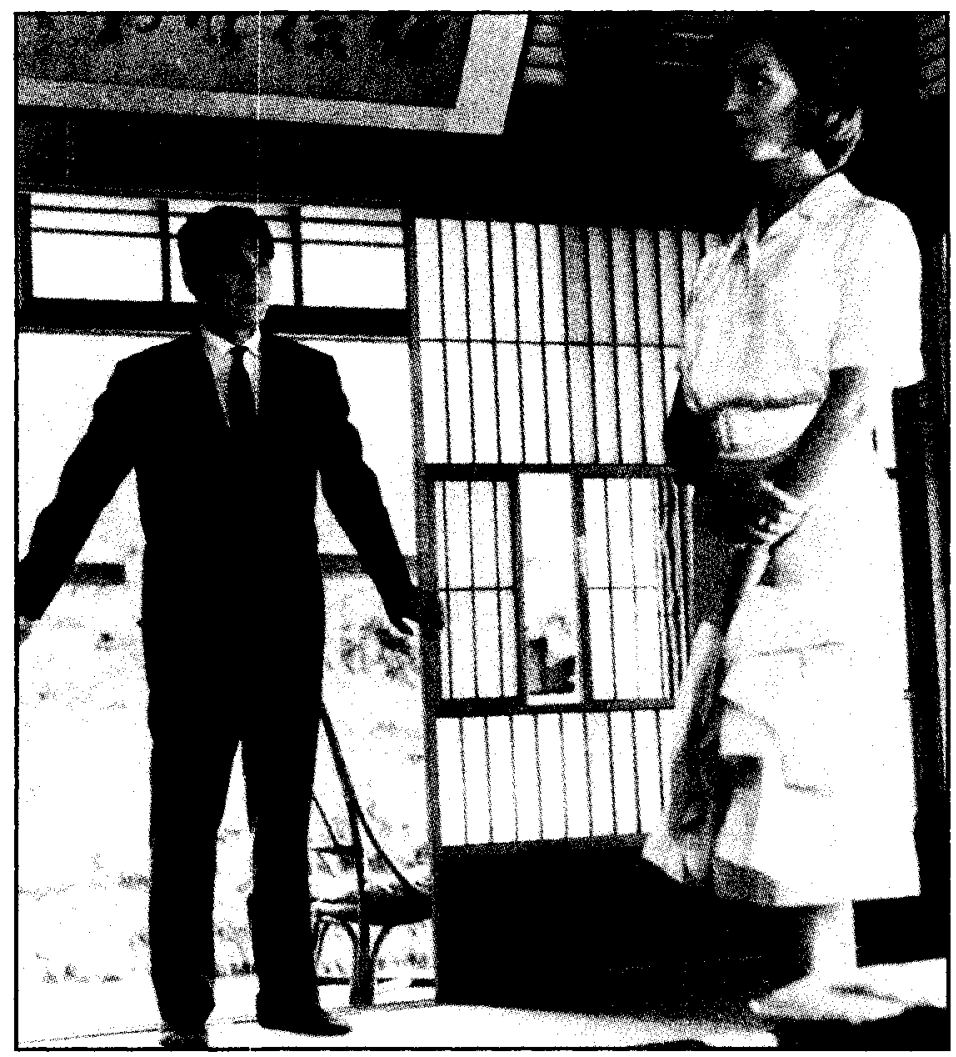

\section{Hiroshima mon amour d'Alain Resnais (1959)}

Tandis quielle regarde ses mains, il apparaît brutalement à la place du Japonais, le corps d'un jeune homme, dans la même pose, mais mortuaire, sur le quai d'un fleuve, en plein soleil [...]. Ce jeune homme agonise. Ses mains sont également très belles, ressemblant étonnamment à celles du Japonais (p. 43).

Il y a bien ici anàbasis, retour, retournement, mais sans la perte puisque, dans son émerveillement devant cette seconde passion impossible pour le Japonais, la Française retrouve le souvenir de l'autre, l'Allemand dont elle doit confronter la mort pour pouvoir en oublier la souffrance.

Il n'est pas innocent que cet épisode soit suivi du réveil du Japonais et de leurs ébats heureux sous la douche. Renaissance de l'amour en quelque sorte.

Fragments orphiques dans Hiroshima mon amour de Marguerite Duras et d'Alain Resnais 
Un second exemple intervient lorsque le regard passionné du Japonais se pose, place de la Paix, sur la Française endormie après la séance de tournage. Ce regard la réveille et la livre de nouveau à la passion alors qu'elle avait exprimé le désir de le quitter définitivement. L'épisode qui suit dans sa maison à lui représente un moment d'intimité accrue entre les deux protagonistes. Dans ces deux exemples, il est intéressant de voir comment chacun des amants revêt tour à tour le rôle orphique. D'où cet enchaînement éloquent entre l'image de la Française et celle du Japonais. Alors qu'elle évoque pour lui la mort de son amant de Nevers, elle apparaît dans la cave, de profil, en plan moyen, noyée dans la pénombre, à droite dans le champ, le regard tourné vers la gauche de l'écran. Dans le plan suivant, le Japonais apparaît à son tour dans la même position. La continuité visuelle rend les deux personnages interchangeables. On peut en effet discerner Orphée dans la Française, qui retourne en arrière dans le passé infernal de la Seconde Guerre mondiale, enfoui au plus profond de son inconscient, s'en souvenant pour mieux le perdre dans "l'oubli universel ". Le Japonais, quant à lui, devient le double de l'Allemand lorsque, noyé dans des éclairages sombres, il déclare: "[...] je suis mort?" (p. 87) ou encore lorsque ses mains apparaissent en plan rapproché tandis que Riva évoque le goût du sang de son amant assassiné. Il veut ainsi devenir catalyseur, psychanalyste, opérateur de transfert afin de susciter la confidence. Il joue donc un rôle orphique lorsqu'il cherche désespérément à retrouver la petite fille de Nevers, afin de mieux cerner son amante du présent: il a en effet choisi de la questionner sur Nevers parce que "C'est là, il me semble l'avoir compris, que tu es si jeune... si jeune, que tu n'es encore à personne précisément. Cela me plaît [...] C'est là, il me semble l'avoir compris, que j'ai failli... te perdre... et que j'ai risqué de ne jamais te connaître" (p. 81). Cette absence de fixation du personnage d'Orphée est encore plus troublante dans les deux scènes du miroir. La première traversée symbolique du miroir (seconde partie) se passe lorsque la Française s'habille en infirmière pour le tournage du film sur la paix. Alors qu'elle se déguise et se regarde dans la glace débute le questionnement du Japonais sur son passé, questionnement auquel elle répond avec résistance: 


\section{LUI}

C'est un joli mot français, Nevers.

Elle ne répond pas tout de suite.

ELLE

C'est un mot comme un autre. Comme la ville.

$$
\text { Elle séloigne (p. 49). }
$$

La seconde traversée (cinquième partie) arrive à la fin du transfert et des aveux de la Française. De nouveau, la Française se regarde dans la glace, où elle s'adresse, dans un dialogue intérieur (voix off), à son amant mort doublement perdu puisqu'elle a osé raconter leur histoire et revivre avec un autre un amour impossible. Dans la scène qui précède cet instant marquant, où regard et parole violent l'interdit du souvenir, la Française est filmée en plans fixes tandis qu'elle s'avance vers la caméra du fond du couloir qui conduit à sa chambre. Le mouvement du personnage est ici totalement contraire au travelling avant de la caméra qui, au début du film, figurait dans le discours filmique le regard glacé, intellectuel de la Française. Il traduit parfaitement dans ce plan, aux éclairages non plus froids, mais contrastés, le regard affectif de celle qui sort de son objectivité aveugle. Celle qui était entrée, déguisée dans le premier miroir, cachée à elle-même dans son refus de voir, ressort du second le regard lavé par l'eau et par les larmes (il est intéressant de rappeler que l'Orphée de Jean Cocteau, qui date de 1950, avait utilisé la traversée du miroir comme passage dans l'au-delà, dans la "zone»). Elle a réappris à voir avec «les yeux du cœur». Elle se précipite alors dans les rues d'un Hiroshima reconstruit et paisiblement endormi. Un autre mythe est esquissé ici : celui du phénix, symbole de la résurrection. De même que la ville japonaise renaît de ses cendres après la bombe, de même la Française, qui s'était crue morte d'amour à Nevers, renaît à l'amour impossible. Elle cumule à la fois le rôle d'Orphée et d'Eurydice et le regard qu'elle s'était interdit sur son passé et qu'elle lui a finalement porté la sauve. Mais ce sauvetage est bien fragile et de courte durée. Lorsque rejointe par le Japonais, la Française répète, comme pour se convaincre, qu'elle restera à Hiroshima, une plongée l'écrase, vaincue, à droite de l'écran et annonce son revirement soudain lorsqu'elle chasse son amant. 
Après s'être éloigné, il va la suivre et l'anàbasis orphique s'impose alors au spectateur et à la spectatrice tandis qu'il la suit, image distante, souvent à peine distincte, à gauche dans le champ. La Française au premier plan, à droite dans le champ, position où tout personnage s'impose, fuit le Japonais tout en attendant passivement le geste qui lui sera fatal: «Il va venir vers moi, il va me prendre par les épaules. Il m'em-bras-se-ra. Il m'embrassera... et je serai perdue" (p. 114). Mais il ne l'embrassera pas. Elle sortira du champ. Lui, seul, sera finalement abandonné, tandis que la caméra s'accroche au pas de la Française, qui déambule dans les rues. Plus tard, il la rejoint pour leur dernier face à face irrémédiable: "Il l'aborde cette fois de face. C'est la dernière fois. Mais il reste loin d'elle. Elle est désormais intouchable» (p. 116). Pour traduire cette coupure définitive, Resnais sépare les amants en plaçant un autre personnage entre eux (la vieille japonaise à la gare d'Hiroshima), en les isolant dans des plans respectifs, et finalement en rendant la Française, filmée en plans d'ensemble, de plus en plus inaccessible. Comme dans le mythe d'Orphée, ce dernier regard échangé semble fatal et pourtant, il semble que la Française ait déjà décidé de son destin, avant le volte-face du Japonais et non à cause de celui-ci. Est-ce ici une réécriture du mythe au féminin? Elle a décidé de la séparation grâce à sa prise de conscience de l'oubli de Nevers.

Quant au sparàgmos, le démembrement, il ne s'inscrit pas de façon impérative, mais semble affleurer dans le scénario, servant de toile de fond à la petite histoire et à la Grande Histoire. Brunel, dans le Dictionnaire des mythes littéraires, rappelle qu'Orphée, après la seconde mort d'Eurydice et son passage par les enfers, se révèle beaucoup plus fragile et que son charme, la puissance de son chant sont remis en question. Il existe plusieurs traditions anciennes de la mort du héros mythologique et la plus répandue serait celle de son assassinat par les Ménades de Thrace sur le mont Pangée: celles-ci, jalouses, n'ont pu supporter le deuil de l'inconsolé, et elles l'ont mis en pièces ${ }^{3}$.

La trace la plus évidente du mythème nous paraît être lorsque, pendant la Libération, la Française est tirée par les cheveux, arrachée au corps de son amant mort et finalement 
tondue par les résistants. "Le bruit des ciseaux sur la tête me laissa dans une totale indifférence. Quand ce fut fait, un homme d'une trentaine d'années m'emmena dans les rues. Ils furent six à m'entourer. Ils chantaient. Je n'éprouvais rien" (p. 149). Le démembrement paraît également explicite au début du film lorsque le spectateur / la spectatrice ne voit pas immédiatement les deux amants. Ainsi, dans la première scène, des corps fragmentés s'entrelacent dans une pénombre telle qu'il est difficile de les identifier, mais la sueur qui perle sur le grain de leur peau évoque à la fois "la rosée de la mort atomique" et les "sueurs de l'amour accompli» (p. 10).

Finalement la petite histoire est orchestrée par la Grande Histoire illustrée par les images documentaires d'un Hiroshima atomisé, mutilé (p. 30). Dès le départ, amour et mort sont inextricablement liés et la Française nous le rappelle tandis que ses propos passionnés et lancinants "Tu me tues, tu me fais du bien " ponctuent la trame sonore. À la fin du film, elle s'adresse en voix off à l'amant mort: "Dévore-moi, déforme-moi. " Cette insistante requête s'adresse aussi à l'amant japonais comme le prouve le montage parallèle qui crée d'étroites correspondances entre Hiroshima et Nevers dont les images viennent interrompre l'errance de l'héröine dans les rues de la ville japonaise. Les deux amants fonctionnent donc comme doubles dans la vie de la Française. Leurs cleux images, nous l'avons précisé, se mélangent de façon hallucinante suggérant que l'amour du mort survit et que l'amour du vivant est voué à la mort: "Par cette étrange osmose, la vitalité d'un survivant de la catastrophe d'Hiroshima se trouve voilée d'un sort macabre, alors que la mort définitive de l'autre survit, diaphane, dans la passion meurtrie de la jeune femme» (Kristeva, p. 254). Cette osmose obsédante s'inscrit, nous l'avons vu, dans les leitmotive du texte, de l'image, comme ceux des éclairages expressionnistes. Les thèmes musicaux créent aussi le même effet de simultanéité: la musique japonaise servant de toile de fond aux événements de Nevers tandis que les motifs musicaux liés à Nevers sont présents dans les scènes à Hiroshima.

Il reste à évoquer la symétrie des deux fleuves: la Loire et la rivière Ota; celle des deux chats: celui, noir, de la cave de 
Nevers, dont les yeux se confondent avec ceux de la Française folle et le chat blanc de la place de la Paix, endormi paisiblement aux pieds de Riva. Il est clair que ces images miroirs créent, tout comme les leitmotive précédemment identifiés, la «[...] syntaxe musicale du film, [qui est] une syntaxe d'équivalences" (Ruwet, cité dans Jakobson, p. 99). Elles servent ainsi la structure même du scénario, qui rappelle celle du second mythème. Chacune comporte ce que Julia Kristeva nomme à propos de l'œuvre durassienne "la réduplication" ou "la répétition bloquée " (p. 254). Cette réduplication est constituée dans le mythe par la seconde chance donnée à Orphée de retrouver Eurydice, elle est bloquée puisqu'elle mène à la perte de l'être aimé.

En conclusion, comme Orphée, la Française d'Hiroshima mon amour a droit à une seconde chance, à un second amour impossible. Comme Orphée, elle perdra cette chance, en prenant conscience de l'oubli universel dans lequel elle engloutira Nevers et Hiroshima. Parallèlement, le cinéaste noie les deux personnages dans un fondu au noir qui clôt le film. Toutefois, cette prise de conscience de Riva, loin de se solder par un échec, paraît au contraire déboucher sur une lucidité, un pouvoir de décision, une liberté qui caractérisent son évolution à la fin du film et qui semblent être un autre exemple de réécriture du mythe au féminin. Indubitablement, elle retrouvera le goût de l'amour interdit, car elle est redevenue disponible. La Française qui nomme son amant japonais Hiroshima n'est plus la même femme qui, au début du film, observait la vie d'un regard distant, glacé, comme en témoignent les éclairages. Tandis qu'elle le regarde, elle est baignée dans des jeux d'ombre et de lumière figurant, par-delà l'affrontement de la vie et de la mort, une renaissance possible. Mais, de même que la reconstruction d'Hiroshima est précaire, de même la résurrection de "la petite coureuse de Nevers» est fragile: phénix atomisé qui se serait brûlé les plumes...

Université Saint Mary's et Université Mount Saint Vincent 


\section{NOTES}

1 Le premier mythème relate l'expédition de Jason, en quête de la Toison d'or, à laquelle Orphée participa. Le second mythème raconte comment Orphée descendit chez Hadès, afin de ramener son épouse Euridyce dans le monde des vivants et comment il échoua dans son entreprise, pour s'être retourné vers elle durant le voyage du retour. Le troisième mythème, enfin, explique comment Dionysos, qu'Orphée vénérait plus qu'Apollon, lâcha sur lui ses Ménades (femmes enragées), qui le mirent en pièces. Toutefois, les Muses recueillirent ses membres dispersés et le déposèrent dans une tombe: sa tête échoua sur l'île de Lesbos.

2 Il faut bien sûr érnettre ici une réserve: Nevers et Hiroshima ne peuvent être évalués et comparés que toutes proportions gardées. Voici le commentaire qu'en fait Resnais lui-même: "We contrast the immense, monstruous, incredible aspect of Hiroshima with the tiny little story of Nevers which to us is reflected through Hiroshima as the glimmer of a candle is magnified and reversed by a lens " (cité dans James Reid, The Great French Films, Secaucus: The Citadel Press, 1983, p. 171-175).

3 Edith Hamilton ajoute même dans La Mythologie (Paris : Marabout, 1978): "Elles jetèrent sa tête dans l'Hèbre et les caux du fleuve la portèrent jusqu'aux abords de l'île de Lesbos. Les Muses la trouvèrent et lui donnèrent une sépulture dans le sanctuaire de l'île, elles rassemblèrent ses membres épars et les déposèrent dans une tombe au pied du mont Olympe et là, jusqu’à ce jour, le chant des rossignols se fait entendre plus doux que partout ailleurs" (p. 123).

\section{OUVRAGES CITÉS}

Borgomano, Madeleine. L'Écriture filmique de Marguerite Duras. Paris: Albatros, 1985.

Brunel, Pierre. Dictionnaire des mythes littéraires. Paris: Du Rocher, 1988.

Chevalier, Jean et Alain Gheerbrant. Dictionnaire des symboles. Paris: Robert Laffont / Jupiter, 1982.

Duras, Marguerite. Hiroshima mon amour. Paris: Gallimard, 1960.

Hamilton, Edith. La Mythologie. Paris: Marabour, 1978.

Jakobson, Roman. Essais de linguistique générale, tome II. Paris: Minuit, 1973.

Kristeva, Julia. Soleil noir. Dépression et mélancolie. Paris: Gallimard, 1987.

Lévi-Strauss, Claude. La Pensée sauvage. Paris: Plon, 1962.

Reid, James. The Great French Films. Secaucus: The Citadel Press, 1983.

Resnais, Alain. "Un cinéaste stö̈cien. Interview d'Alain Resnais". Esprit, vol. 28 n" 285 (1960), p. 934-945.

Robinson, W.R. "If You Don't See, You Are Dead". Contemporary Films / McGraw Hill, vol. 2, n" 3 (1972), p. 20-31. 